\title{
Explorando a Trajetória Espácio-Temporal da Representação Dinâmica de Projéteis
}

\author{
Exploring the Spatiotemporal Trajectory \\ of the Dynamic Representation of Projectiles
}

\author{
Nuno Alexandre De Sá Teixeira* \& Armando Mónica Oliveira \\ Universidade de Coimbra, Coimbra, Distrito de Coimbra, Portugal
}

\begin{abstract}
Resumo
Quando é mostrado a observadores humanos um objeto em movimento horizontal que desaparece subitamente, e se instruídos a indicar o local de desaparecimento, emerge sistematicamente um erro para diante na direção do movimento (Desfasamento $\mathrm{M}$ ) e para baixo na direção da gravidade (Desfasamento O). Ainda que diversos determinantes do fenómeno estejam bem documentados, pouco é ainda sabido acerca do seu curso temporal. O presente estudo procura preencher esta lacuna. Objetos descrevendo movimentos horizontais foram mostrados a participantes instruídos a indicarem o seu local de desaparecimento, usando ou um mouse ou um ponteiro (num ecrã táctil), após um intervalo de retenção variado. Os resultados revelaram uma trajetória ordenada para os erros de localização em função do tempo, passível de ser descrita em duas fases - numa primeira, até cerca de $300 \mathrm{ms,}$ os erros progrediram para diante sem qualquer desfasamento vertical; após os $300 \mathrm{~ms}$ o erro tende a aumentar na direção descendente sem qualquer incremento horizontal. Paralelos deste padrão com tarefas de física intuitiva (Física de Road Runner) e antecedentes na História Pré-Galilaica da Física são referidos e os resultados discutidos no âmbito de uma representação implícita de invariantes físicos na perceção de eventos dinâmicos.

Palavras-chave: Percepção do movimento, Física Intuitiva, Momento Representacional.
\end{abstract}

\begin{abstract}
When human observers are shown a horizontally moving target which suddenly disappears and they are further instructed to locate its vanishing position, both forward in the direction of motion (M Displacement) and downward in the direction of gravity (O Displacement), errors of localization typically occur. Though several determinants of those errors have been ascertained, little is known regarding their time course. The present study attempts to fill this gap. Horizontally moving targets were presented and participants instructed to locate their vanishing position, either via a mouse or a pointer (on a touch screen) after a variable time delay. Outcomes revealed an orderly time-dependent trajectory of errors being describable in two stages - during the first $300 \mathrm{~ms}$, the errors increased in the direction of motion with a constant vertical error; after $300 \mathrm{~ms}$ the downward error increased with no further horizontal displacement. Similarities between this pattern and reported results from the Intuitive Physics (Road Runner Physics) and the History of Ancient Physics are noticed and discussed under the notion of an implicit representation of physical invariants in the perception of dynamic events. Keywords: Motion perception, Intuitive Physics, representational momentum, representational gravity.
\end{abstract}

Uma condição óbvia para a sobrevivência e adaptação, quer enquanto indivíduos quer enquanto espécie, refere-se à apreensão e ação do e no mundo físico, no qual a variável tempo emerge como uma das mais relevantes. Existimos essencialmente como seres dotados de mobilidade e frequentemente interagimos com outros seres ou

\footnotetext{
* Endereço para correspondência: Instituto de Psicologia Cognitiva, Universidade de Coimbra, Rua do Colégio Novo, Apartado, 6153, Coimbra, Portugal 3001-802. E-mail: nuno_desateixeira@fpce.uc.pt

O presente trabalho foi financiado pela Fundação para a Ciência e Tecnologia no âmbito dos projetos SFRH/ BD/36067/2007 e POCI/PSI/60769/2004.
}

objetos também eles móveis, em eventos que se desdobram ao longo do tempo. Neste sentido, não resulta estranha a observação de que os seres humanos se mostram particularmente sensíveis à informação relativa a transformações/ alterações dinâmicas no seu meio ambiente (e.g., Gibson, 1986; Johansson, 1975).

A relevância deste ponto veio a ser sublinhada na noção de Representações Dinâmicas (Freyd, 1987), a qual sugere que a própria informação temporal é não só intrínseca (analogicamente) como necessária nas e para as representações mentais. De resto, o sucesso amplamente constatável das nossas interações percetivo-motoras perante eventos físicos sugere que as variáveis temporais 
são parte integrante dos ciclos percetivos e respetiva coordenação sensorimotora (e.g. Bertamini, Spooner, \& Hecht, 2004). Um fenómeno que parece captar simultaneamente aspetos representacionais de noções dinâmicas (Freyd, 1992, 1993) bem como particularidades do ciclo perceção-ação (Kerzel \& Gegenfurtner, 2003) veio a ficar conhecido como Momento Representacional ${ }^{1}$.

\section{O Momento Representacional}

Perante um objeto móvel que desaparece subitamente, e se instruídas a indicar o local do desaparecimento, as pessoas tendem sistematicamente a indicar um ponto desfasado para diante na direção do movimento (Freyd \& Finke, 1984).

No paradigma original, os participantes eram expostos a uma sucessão de três retângulos, espaçados a intervalos regulares de $250 \mathrm{~ms}$, e com sucessivas orientações congruentes com um movimento de rotação. Após a apresentação do último retângulo, era apresentado aos participantes um quarto retângulo (sonda mnésica) com uma orientação igual ou mais ou menos rodada em relação ao último retângulo da sequência indutora. Aos participantes era pedido que indicassem se a sonda mnésica se encontrava na mesma orientação que o último retângulo da sequência ou numa orientação diferente (paradigma igual-diferente). Os resultados típicos nesta situação traduzem-se numa probabilidade mais elevada de se aceitar como "igual" um retângulo que, na realidade, ocupa uma orientação mais rodada na direção do movimento. De acordo com Freyd e Finke (1984), este desfasamento revelaria um análogo representacional do momento (quantidade de movimento de um objeto móvel; dado pelo produto da sua massa com a velocidade) e inércia (tendência a manter ou o estado de movimento ou de repouso). Isto é, os eventos seriam representados internamente com propriedades análogas às de um evento físico. Na situação descrita, e perante o súbito desaparecimento do estímulo, a representação visual, dotada ela mesma de um análogo do momento e da inércia, tendia a prosseguir o seu "movimento", resultando num desfasamento mnésico da última posição observada - Momento Representacional (MR).

Em congruência com esta interpretação, verificou-se que o desfasamento emergia somente quando a sequência implicava um movimento e que alterações do objeto visualizado (e.g., da sua forma ou textura) introduzidas ao longo da sequência indutora (Kelly \& Freyd, 1987)

${ }^{1} \mathrm{O}$ Momento Representacional é um de vários fenómenos de localização espacial. Neste sentido, partilha similaridades teóricas e empíricas com fenómenos tidos como de baixo nível, como o sejam o efeito Fröhlich (e.g., Müsseler, Stork, \& Kerzel, 2002) e efeito flash-lag (Müsseler et al., 2002; no entanto, ver Nagai et al., 2010), fenómenos sensíveis a determinantes de alto-nível como efeito On-Set Repulsion (Actis-Grosso \& Stucchi, 2003) e Boundary extension (Munger, Owens, \& Conway, 2005), e mesmo fenómenos declaradamente cognitivos como Tempo de Colisão (Gray \& Thornton, 2001) e resultados de física ingénua (e.g., Hubbard, 2005). enfraqueciam a sua magnitude. De igual forma, o desfasamento mostrou-se proporcional à velocidade final do objeto (Freyd \& Finke, 1985), independentemente dos padrões de aceleração/desaceleração verificados durante a sequência (Finke, Freyd, \& Shyi, 1986). De resto, a presença de movimento real parece não ser necessária desde que o estímulo o implique de alguma forma - caso de fotografias ou desenhos estáticos que impliquem movimento, os quais conduzem a desfasamentos mnésicos em tudo similares ao MR (Freyd, 1983; Freyd, Patzer, \& Cheng, 1988).

Convém sublinhar aqui o carácter "quasi-amodal" do MR. Note-se que este emerge após o desaparecimento do estímulo físico; ou, melhor, a resposta do participante é dada na ausência desse. Este ponto está longe de ser displicente pois é hoje aceite que, aquando da visualização de um evento dinâmico, este é de alguma forma simulado ou emulado representacionalmente (e.g., Grush, 2004). Obviamente, a própria presença de estimulação física constrange a representação do movimento a uma interpretação unívoca - neste sentido, na perceção de movimento, a distinção entre processos top-down e bottom-up é em certa medida mais esbatida pois ambos se conjugam na perceção do evento. Contudo, e dado que numa tarefa típica de MR o alvo já não está acessível à perceção, a resposta do participante depende somente dos mecanismos que se encontravam recrutados para o processamento em linha (online) aquando da visualização do movimento - isto é, a representação do evento (processos top-down). O súbito desaparecimento do objeto móvel implica pois que a resposta do participante dependa somente dessa. Mais que isso, sugere que os invariantes empíricos reportados para o MR revelem na verdade aspetos nomotéticos da própria dinâmica da representação mental, não constrangida, portanto, por uma estimulação visual física, exceto no que se refere à sua mobilização inicial.

Dado que a variável tempo é ela mesma intrínseca e necessária (Freyd, 1987) às Representações Dinâmicas, tais processos deverão estar sujeitos a um curso temporal (Freyd \& Johnson, 1987); isto é, um padrão de evolução em função do tempo que reflete a dinâmica inerente à representação. Num sentido próprio, descrever o curso temporal do MR equivale pois a estabelecer a mecânica representacional da perceção do movimento.

\section{O Tempo nos Estudos do MR}

Conforme vimos, tanto a noção de Representações Dinâmicas como a noção de um Momento físico internalizado levariam a esperar um efeito da variável tempo sobre a magnitude do MR. Concretizando a analogia, considere-se um objeto móvel ao qual é aplicada uma força contrária ao movimento com o intuito de o interromper. O estado de repouso será atingido tão mais tarde quanto o momento ou quantidade de movimento do objeto (assumindo uma força resistiva constante); além disso, prosseguirá o seu movimento durante algum tempo antes de atingir, por 
fim, o estado de repouso. Se o MR constituir um análogo do momento, um padrão similar deverá ser obtido numa tarefa adequada.

A forma óbvia de testar esta hipótese implica tão-somente manipular o intervalo de retenção-i.e., o tempo que medeia a apresentação do último estímulo da sequência e a apresentação da sonda mnésica. Esta experiência, conduzida por Freyd e Johnson (1987), veio a revelar dados congruentes com essa previsão - a magnitude do MR tende a aumentar com o tempo de retenção até atingir um máximo por volta dos $300 \mathrm{~ms}$ (milissegundos). No âmbito da noção de Representação Dinâmica, este padrão de resultados sugere que, após o término da estimulação e pelo menos até $300 \mathrm{~ms}$ depois, os processos representacionais recrutados para o processamento do movimento mantiveram a sua "dinâmica". Isto é, durante o intervalo de retenção, a representação estaria ainda sob efeitos dinâmicos, inerentes à própria e não obstante a ausência do estímulo físico. Adicionalmente, os dados de Freyd e Johnson (1987) mostram a presença de MR mensurável com intervalos de apenas 10 milissegundos, atestando a rapidez desses mecanismos.

Mais recentemente, Kerzel (2000), impondo aos participantes restrições nos movimentos oculares, verificou, com estímulos de movimento contínuo (i.e., fluído), não só um virtual desaparecimento do Momento Representacional, como uma insensibilidade deste ao intervalo de retenção. Este resultado veio a constituir a base da principal crítica à conceção "representacional" do MR, pelo menos dado um movimento contínuo (por oposição a aparente): o desfasamento na direção do movimento pode implicar não um momento representacional mas sim um momento biofísico da própria mecânica dos movimentos oculares. Dito de outra forma, e perante o desaparecimento súbito do objeto, o movimento de perseguição do olho tende a continuar para além do local de desaparecimento, levando a que os participantes julguem como "igual" um objeto desfasado nessa direção. Esta posição falha em explicar um conjunto considerável de determinantes do MR (e.g., a sua emergência em imagens estáticas, onde não existe movimento de perseguição ocular; efeitos conceptuais obtidos por variações da identidade do alvo²; e.g., Reed \& Vinson, 1996; equivalentes do MR para outras modalidades percetivas: e.g., auditiva ${ }^{3}$, Freyd et al., 1990; Getzmann,

\footnotetext{
${ }^{2}$ Por exemplo, uma representação esquemática de um foguetão mostrada em movimento ascendente conduz a um maior desfasamento para cima do que uma representação geometricamente equiparável de um edifício.

${ }^{3}$ Tem sido hipotetizado que equivalentes do Momento Representacional emergem para qualquer contínuo intensivo. No estudo de Freyd, Kelly e DeKay (1990) era mostrado aos observadores uma sequência de sons ordenada por frequência (e.g., 1261, 1892, $2523 \mathrm{~Hz}$ ). De seguida, eram expostos a um único som (sonda mnésica) e instruídos a indicar se esse seria igual ou diferente do último som da sequência. Os participantes mostravam uma maior probabilidade de aceitar como "igual" um som com uma diferença de cerca de $3.31 \mathrm{~Hz}$ na direção sugerida pela sequência (e.g., $2526.31 \mathrm{~Hz}$ ).
}

2005). Neste sentido, os movimentos oculares parecem ser necessários mas não suficientes para uma explicação global do fenómeno. Contudo, e no caso particular da descrição do seu curso temporal, este resultado nulo veio a ser tomado como decisivo e nenhuma outra experiência sobre o tópico veio a ser conduzida.

\section{Os Componentes Horizontal e Vertical do} Desfasamento e Respostas Motoras de Localização

Com a introdução de modalidades de resposta baseadas na localização motora (e.g., usando um mouse de computador para clicar no local de desaparecimento percebido; por oposição ao paradigma da sonda mnésica; Hubbard \& Bharucha, 1988), os determinantes do MR multiplicaram-se na literatura. Foram, pois, reportados análogos representacionais da fricção (Hubbard, 1995), ímpeto centrípeto (Hubbard, 1996), causalidade mecânica (Hubbard, Blessum, \& Ruppel, 2001) e gravidade (tendência a indicar um ponto desfasado na direção da gravidade; Hubbard, 1990, 1995, 1997, 2001; cf. também Bertamini, 1993).

Efetivamente, o uso de modalidades de resposta de localização espacial possibilita não só o estudo do desfasamento na direção do movimento (Momento Representacional propriamente dito; Desfasamento $\mathrm{M}$ ) como também do desfasamento na direção vertical, ortogonal em relação ao eixo de movimento horizontal (Desfasamento O; Ver Figura 1). Este último componente (Desfasamento O) tem sido consistentemente apontado como uma medida da Gravidade Representacional, i.e., um análogo da gravidade física (Hubbard, 1990, 1995, 1997, 2001).

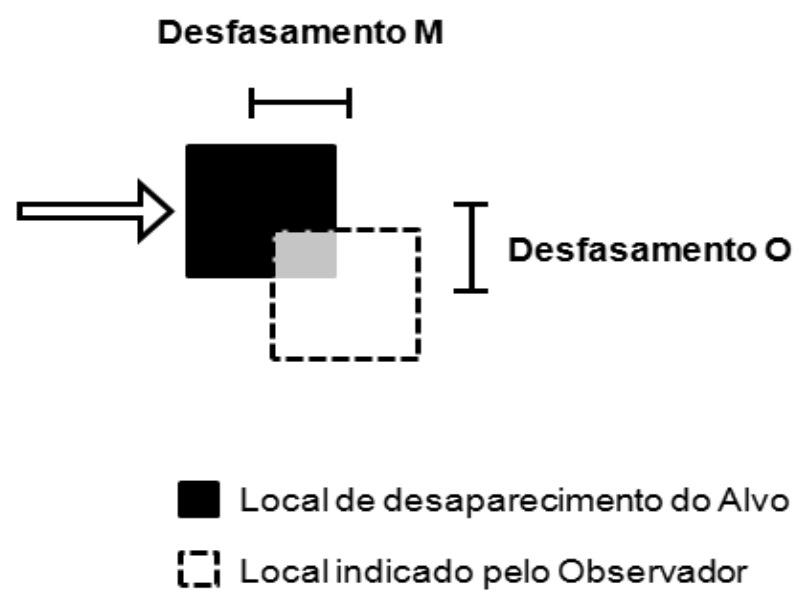

Figura 1. Componentes dimensionais do desfasamento mnésico para a localização de um objeto móvel que desaparece subitamente. $\mathrm{O}$ desfasamento $\mathrm{M}$ traduz a tendência para indicar um ponto na direção do Movimento e é dado pela diferença algébrica entre a coordenada horizontal do ponto indicado pelo observador e a do local físico do desaparecimento. O desfasamento O traduz uma tendência a indicar um ponto desfasado na direção da gravidade - é dado pela diferença entre a coordenada vertical indicada pelo observador e a do local de desaparecimento do alvo. 
Por outro lado, o uso de respostas de localização espacial revelou que os efeitos do constrangimento dos movimentos oculares, atrás discutidos, são contingentes à modalidade de resposta - para respostas de localização, inerentemente motoras, e ao contrário do que ocorria no paradigma da sonda mnésica (ver atrás; Kerzel, 2000), a presença de movimentos oculares revelou-se não necessária para a emergência do fenómeno. Isto é, o constrangimento dos movimentos oculares não parece acarretar qualquer redução na magnitude do desfasamento para estas modalidades (Ashida, 2004; Kerzel, 2005; Kerzel \& Gegenfurtner, 2003). No seu conjunto, estes dados sustentam a necessidade de um envolvimento motor na emergência do MR: seja mediante movimentos oculo-motores, no caso de um juízo percetivo (paradigma da sonda mnésica), ou uma interação motora aquando da resposta (paradigma de localização espacial), ou ambos. De resto, estes resultados vieram a estreitar a já sugerida relação entre o MR e os ciclos de perceção-para-a-ação (Ashida, 2004; Brouwer, Franz, \& Thornton, 2004; Kelly \& Freyd, 1987; Kerzel \& Gegenfurtner, 2003; Zago \& Lacquaniti, 2005). Nomeadamente, reforçaram a sugestão de que o MR seria instrumental na compensação de tempos de latência acumulados ao longo das vias neurais de processamento da informação visual (hipótese de extrapolação do movimento e compensação dos tempos neuronais; Kerzel \& Gegenfurtner, 2003; Nijhawan, 1994, 2008) e de forma a sincronizar as ações motoras com os eventos dinâmicos no mundo físico.

Num sentido similar, e fundado na distinção entre cinemática e dinâmica (Runeson \& Fryckholm, 1983), estudos por nós conduzidos têm suportado a ideia de que a natureza das variáveis (cinemática ou dinâmica) é modulada diferencialmente pelo grau de envolvimento motor em modalidades de resposta de localização espacial. De forma breve, a Cinemática compreende os movimentos propriamente ditos restringindo-se (de forma quase puramente descritiva) a conceitos como velocidade, aceleração, distância, tempo, etc. Já a Dinâmica compreende conceitos de cariz explicativo e com recurso a conceitos de "Força" (e.g., momento, fricção, gravidade, etc.). Do ponto de vista de um observador, a distinção adquire significância se notarmos que os aspetos cinemáticos são dados por si mesmos, pela sua própria existência; i.e., podem ser percecionados independentemente de uma estrutura causal de forças. Contrariamente, uma apreensão de aspetos Dinâmicos requer uma estrutura de significação imposta no próprio movimento, ou na relação unívoca com a cinemática (como no caso do Princípio da Especificação Cinemática da Dinâmica; Runeson \& Frykholm, 1983) ou por dados adicionais independentes dessa (e.g., pela identificação do agente do movimento [natural vs causado], pelo reconhecimento das propriedades do alvo [animacidade, peso/massa, forma, propulsão, etc.]). No caso do MR tem emergido um padrão sistemático no qual a importância do efeito de variáveis de cariz dinâmico é reduzido para modalidades de resposta diretas (e.g., toque manual num ecrã táctil) por comparação com modalidades indiretas ou mediadas (e.g., Mouse e Teclado; e.g., De Sá Teixeira, Oliveira, \& Amorim, 2010).

\section{Revisitando o Papel do Tempo no MR}

Posto isto, é legítimo reavaliar o curso temporal do MR. Por um lado, o papel dos movimentos oculares não se mostra determinante perante o uso de paradigmas de localização espacial - descrever o curso temporal do desfasamento nestas modalidades de resposta surge assim como um dado importante no esclarecimento do fenómeno. Por outro, nenhum estudo procurou ainda averiguar o curso temporal da Gravidade Representacional (e.g., pelo desfasamento-O), um resultado que, uma vez mais, depende do uso de respostas de localização espacial.

Ainda que, a rigor, o tempo seja uma variável de cariz cinemático, neste contexto assume um pendor representacional (o tempo da transformação/evolução da representação mental) e, como tal, diferencia-se de variáveis como a velocidade e distância, as quais, pela própria manipulação constrangem a representação ao que é percecionado pelo participante. Pelo contrário, o tempo de retenção, assumindo um carácter amodal (no sentido em que o objeto indutor já não se encontra presente), concilia à sua natureza fisicamente cinemática (manipulação objetiva do intervalo de retenção) propriedades "representacionalmente" dinâmicas - ocorre ao nível da representação cognitiva, da qual é intrínseca, e deverá, portanto, traduzir o seu funcionamento interno.

Por fim, e como as respostas de localização espacial possibilitam a medida simultânea de dois componentes (Desfasamento M e O), torna-se possível averiguar o desdobramento temporal das sucessivas "localizações" num mapa bi-dimensional, obtendo assim uma trajetória representacional.

\section{Método}

\section{Participantes}

Quarenta e oito estudantes (41 do sexo feminino) da Universidade de Coimbra, com idade média de 19,94 anos $(D P=3,87)$, participaram na experiência. Todos possuíam visão normal ou corrigida para o normal e desconheciam os propósitos do estudo.

\section{Estímulos}

Um conjunto de animações (.avi a 40 fps) que mostravam um quadrado negro com $30 \mathrm{px}$ (pixels; $30 \mathrm{px} \approx$ $1 \mathrm{~cm})$ de lado a viajar a velocidades constantes de 180 , 300,420 ou $540 \mathrm{px} / \mathrm{s}$. Cada quadrado (alvo) emergia do extremo esquerdo ou direito do ecrã, desaparecendo após cobrir uma distância total de 536, 593 ou 650 px. Todas as animações foram criadas com o software Interactive Physics 2004 e editadas com o VirtualDub. A experiência 
Teixeira, N. A. A. \& Oliveira, A. M. (2013). Explorando a Trajetória Espácio-Temporal da Representação Dinâmica de Projéteis.

foi implementada no ambiente Super Lab 4.0, com o qual eram apresentados os estímulos, recolhidas as respostas e aleatorizados os ensaios. Os estímulos foram apresentados num computador pessoal equipado com um ecrã plano táctil, com uma taxa de refrescamento de $60 \mathrm{~Hz}$ e resolução de ecrã de $1024 \times 768$ px. Metade dos participantes fornecia as suas respostas usando um mouse sem fios com o qual controlava um cursor em forma de cruz $(+)$ mostrado no ecrã (condição Mouse); a outra metade tocava diretamente no ecrã usando um ponteiro (condição Ponteiro).

\section{Desenho e Procedimento}

A experiência obedeceu a um desenho fatorial de medidas repetidas 4 (Velocidade) $\times 3$ (Distância) $\times 5$ (Intervalo de retenção) $\times 2$ (Direção do movimento), com 5 replicações. Os participantes sentavam-se a cerca de 60 $\mathrm{cm}$ do ecrã, sem restrições de movimentos oculares ou de cabeça, mas instruídos a manterem uma postura estável e constante ao longo da experiência (o seguimento das instruções foi monitorizado por observação informal). Os participantes eram instruídos a observar atentamente o movimento dos quadrados e, quando ouvissem um som (beep; condição Ponteiro) ou quando o cursor do mouse (+) surgisse no ecrã (condição Mouse), a indicarem, o mais precisamente possível, o local onde o alvo havia sido visto pela última vez (i.e., o seu local de desaparecimento). O som (Ponteiro) ou o cursor (Mouse) podiam aparecer $0,150,300,450$ ou 600 milissegundos após o desaparecimento do alvo. De forma a garantir que a resposta era iniciada somente após o intervalo de retenção, os movimentos do mouse foram monitorizados e, perante a deteção de um movimento antes do aparecimento do cursor, esse ensaio era repetido algures durante a sessão experimental. De forma similar, e para o caso do Ponteiro, cada ensaio era iniciado com o premir de um botão numa caixa de respostas, usando a mesma mão que segurava o ponteiro com o qual deveria ser dada a resposta. Esse botão deveria permanecer premido até à apresentação do som de início da resposta. Caso o botão fosse solto antes desse evento, o ensaio era repetido mais tarde (Ver Figura 2). Alguns ensaios de treino antecediam a experiência propriamente dita, de forma a garantir a compreensão da tarefa. O procedimento respeitou a declaração de Helsinque quanto à experimentação em seres humanos.

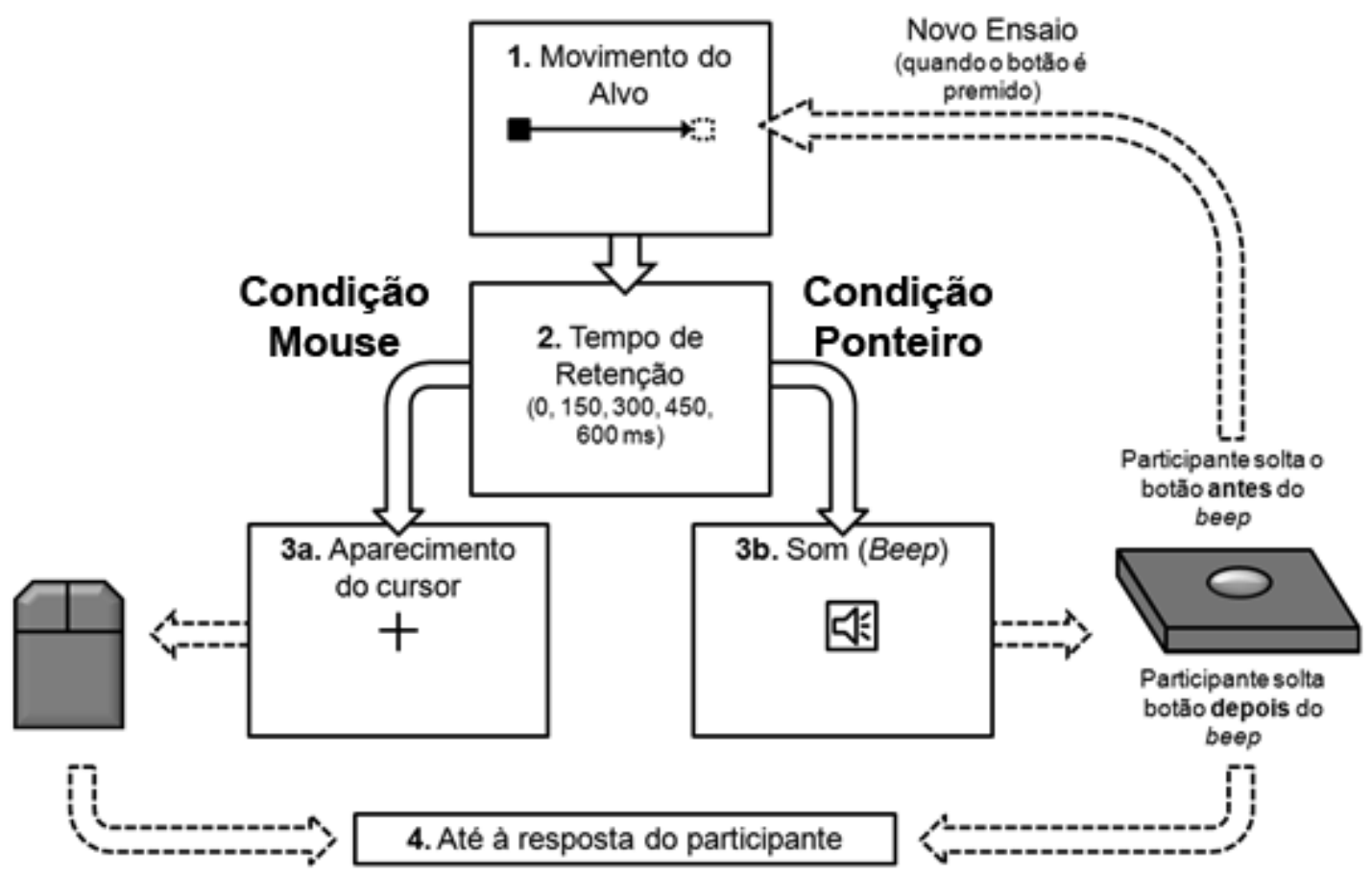

Figura 2. Representação esquemática da sequência de eventos da experiência.

\section{Resultados}

O desfasamento M (no eixo do Movimento; Momento Representacional/MR) foi calculado pela diferença algébrica entre as coordenadas $x$ do local no ecrã indicado pelos participantes e as mesmas coordenadas para o centro geométrico do alvo no último diapositivo de cada animação. Um valor positivo denuncia um desfasamento na direção do movimento e um valor negativo um desfasamento na direção oposta à do movimento. $\mathrm{O}$ desfasamento $\mathrm{O}$ (Ortogonal ao eixo do movimento; Gravidade Representacional) foi calculado pela diferença entre as coordenadas $y$ do local do ecrã indicado pelo participante e as mesmas coordenadas referente ao centro geométrico do alvo no último diapositivo. Desta forma, um valor negativo indica um desfasamento na direção descendente (no sentido 
da gravidade) e um valor positivo um desfasamento na direção ascendente. Os dados assim obtidos foram sujeitos a ANOVAs mistas tendo como fator entre-sujeitos a modalidade de resposta. Nos casos em que se verificou uma violação do pressuposto da esfericidade, usou-se a correção Greenhouse-Geisser para os graus de liberdade.

\section{Desfasamento $M$}

No que se refere ao desfasamento na direção do movimento (Momento Representacional), obtiveram-se efeitos principais da Velocidade, $F(1.793,82,497)=49,393$, MQE $=935,468, p<0,01, \eta_{\mathrm{p}}^{2}=0,52$ (com a magnitude do desfasamento aumentando proporcionalmente à velocidade), e Tempo de Retenção, $F(2.46,113,142)=12,311, \mathrm{MQE}=$ $517,483, p<0,01, \eta_{\mathrm{p}}^{2}=0,21$ (ver Figura 3). Por outro lado, a modalidade de resposta apresentou uma interação significativa com o Intervalo de Retenção, $F(2.46,113,142)$ $=11,271, \mathrm{MQE}=517,483, p<0,01, \eta_{\mathrm{p}}^{2}=0,2$. ANOVAs efectuadas separadamente para as modalidades de resposta revelaram um efeito do Intervalo de Retenção com o uso do Mouse, $F(2.922,67,198)=19,393 \mathrm{MQE}=486,331, p$ $<0,01, \eta_{\mathrm{p}}^{2}=0,46$, mas não do Ponteiro $(p>0,05)$.

\section{Desfasamento $O$}

Para o desfasamento ortogonal à direção do movimento (Gravidade Representacional), verificou-se que a velocidade do alvo não atingiu a significância estatística $(p>$ $0,05)$. O Tempo de Retenção atingiu um efeito principal significativo, $F(4,184)=7,432, \mathrm{MQE}=28,289, p<0,05$, $\eta_{\mathrm{p}}^{2}=0,14$ (ver Figura 3). ANOVAs de medidas repetidas aplicadas separadamente para as condições revelou que o Tempo de Retenção atingiu um efeito estatisticamente significativo na modalidade Mouse, $F(2.765,63,584)=$ $10,924, \mathrm{MQE}=29,778, p<0,05, \eta_{\mathrm{p}}^{2}=0,32$, mas não na modalidade Ponteiro $(p>0,05)$.

\section{A Trajetória Bi-Dimensional do Desfasamento}

Dada a bi-dimensionalidade das respostas dos participantes, é possível apresentar os dados num "mapa" de desfasamentos tendo como eixo horizontal o componente $\mathrm{M}$ e como eixo vertical o componente $\mathrm{O}$ (ver Figura 3). Num sentido quase literal, este traduz a topografia da representação que subjaz à perceção de eventos dinâmicos. Os círculos a negro traduzem os desfasamentos bi-dimensionais obtidos com o uso do Mouse em função dos Tempos de Retenção. Os círculos a branco representam a mesma informação para a condição Ponteiro. É possível verificar que nenhum padrão emerge na modalidade Ponteiro - os pontos tendem a agrupar-se sensivelmente na mesma localização. Contudo, no caso do uso do Mouse é evidente uma "trajetória" ordenada, na qual a representação do alvo tende a "mover-se" para diante até cerca dos 150/300 ms, sem qualquer desfasamento descendente. Após os $300 \mathrm{~ms}$ é possível constatar uma "queda" progressiva da localização mnésica do alvo, sem qualquer incremento na sua posição horizontal.

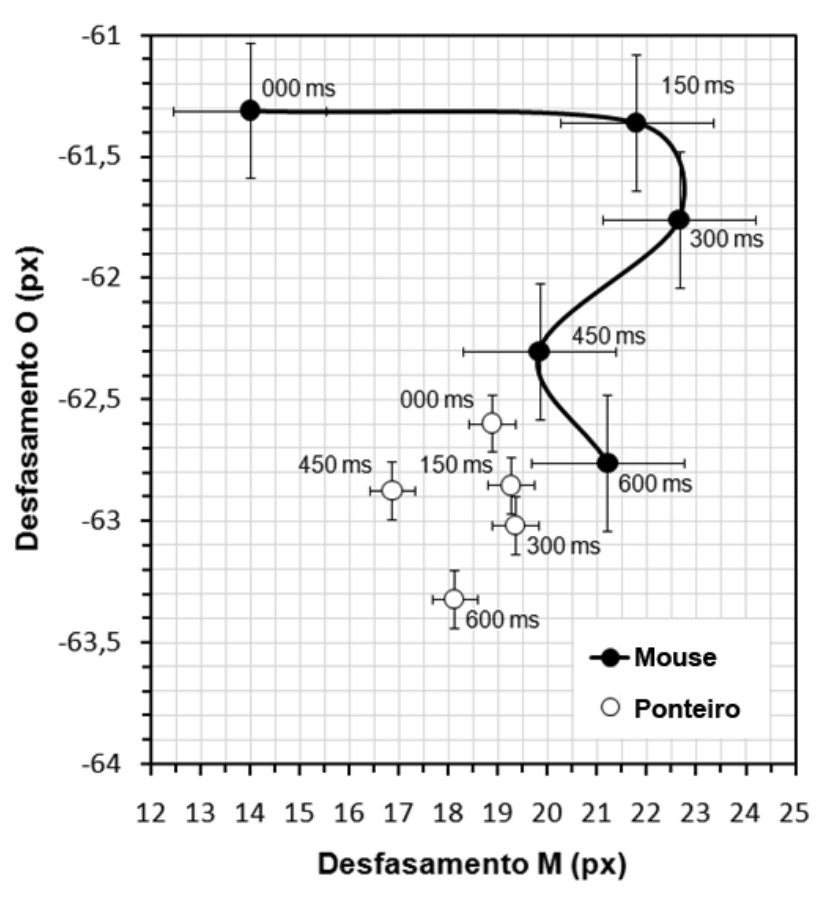

Figura 3. Mapa dos desfasamentos em pixéis obtidos em função do tempo, tendo como eixo horizontal os valores do componente $\mathrm{M}$ e como eixo vertical os valores do componente O. O local de desaparecimento do alvo situa-se na origem (ponto 0,0 ). Os círculos indicam as localizações indicadas pelos participantes em função dos tempos de retenção (rótulos). Os círculos negros referem-se à condição Mouse e os círculos brancos à condição Ponteiro. As barras de erro traduzem o erro-padrão da média para ambos os componentes.

\section{Discussão e Conclusão}

Os dados apresentados revelam, antes de mais, uma dissociação entre as duas modalidades de resposta motora (Mouse e Ponteiro) no que se refere ao curso temporal. A saber, a condição Ponteiro mostrou-se insensível ao tempo de retenção. Este dado sugere um envolvimento distinto dos circuitos de perceção-para-ação e perceção-para-reconhecimento (Milner \& Goodale, 1995) no MR, sendo o primeiro relativamente insensível a aspetos representacionais de alto-nível, de cariz mais cognitivo. Esta modulação dos padrões contingente ao grau de envolvimento motor concorre com resultados previamente obtidos (De Sá Teixeira et al., 2010).

No que se refere ao curso temporal do desfasamento na modalidade Mouse obteve-se um padrão passível de ser descrito por um processo desdobrado em duas fases: (a) até cerca dos $300 \mathrm{~ms}$ a representação do alvo prosseguiu o seu "movimento" para diante, revelando-se relativamente insensível à influência gravitacional; (b) após os 300 ms, a representação do alvo tende a "cair" na direção da gravidade.

Este padrão de resultados, nunca reportado para tarefas de localização espacial, encontra-se bem documentado na literatura sobre Física Intuitiva (Gentner, 2002; Mc- 
Closkey, 1983), sob o nome de Física de Road Runner (em Português, Papaléguas e Coiote). Nesses desenhos animados é, com efeito, frequentemente representado um personagem (invariavelmente o Wile E. Coyote) a ser lançado horizontalmente de um precipício até ao momento em que, perdendo por completo a velocidade, cai numa trajetória reta em direção ao solo. Obviamente, este padrão de movimentos viola o postulado normativo dado pela Físicaseja a Física Newtoniana, de acordo com a qual um projétil segue uma trajetória parabólica, seja a Física Relativista de Einstein que prevê uma trajetória hiperbólica. Encontra, porém, na Física Medieval (Pré-Galilaica) proto-científica um antecedente, fundado na noção de ímpeto - força motriz transferida a um projétil aquando do seu lançamento, que se desvanece com o próprio movimento ao longo do tempo. Por exemplo, Jean Buridan (1300-1358; cf. e.g., McCloskey, 1983) argumentou que o movimento de um projétil se manteria até que o ímpeto se dissipasse, ficando esse sujeito ao comportamento comum dos graves. A atestar que tal proposta era relativamente aceite na época, Paulus Puchner, em 1577 (cf. Hecht \& Bertamini, 2000), elabora uma análise das trajetórias desenhadas pelas balas de canhão após o disparo, de forma a servir de auxílio à artilharia saxónica (ver Figura 4). Neste, a trajetória dos projéteis é representada seguindo um percurso em duas fases (três se se considerar a fase curva intermédia; ver Hecht, 2001): num primeiro momento a bala seguiria um percurso em linha reta até uma completa dissipação do ímpeto, após o qual cairia na vertical, uma vez mais em linha reta (a rigor, estas trajetórias terão sido derivadas da teoria Aristotélica e, em certos detalhes, opõe-se ao previsto por uma física do ímpeto na sua forma mais pura; especificamente, a fase em curva sugere desde logo que o projétil esteve sempre sujeito à influência gravitacional e não somente ao ímpeto; contudo, para os presentes propósitos bastará sublinhar o comportamento global sugerido para os projéteis; cf. Hecht, 2001). Esta clivagem entre movimentos/tendências "naturais", como o seja a gravidade (eixo vertical), e movimentos provocados, i.e., projéteis (eixo horizontal), distinção peripatética corrente na época medieval (Koyré, 1992; Jammer, 1957), parece subjazer aos dados empíricos obtidos (confrontar Figura 4 com Figura 3): a sensibilidade da representação do alvo a uma influência natural (Gravidade Representacional) emerge somente aquando da dissipação do seu impulso (ímpeto) causado (Momento Representacional).

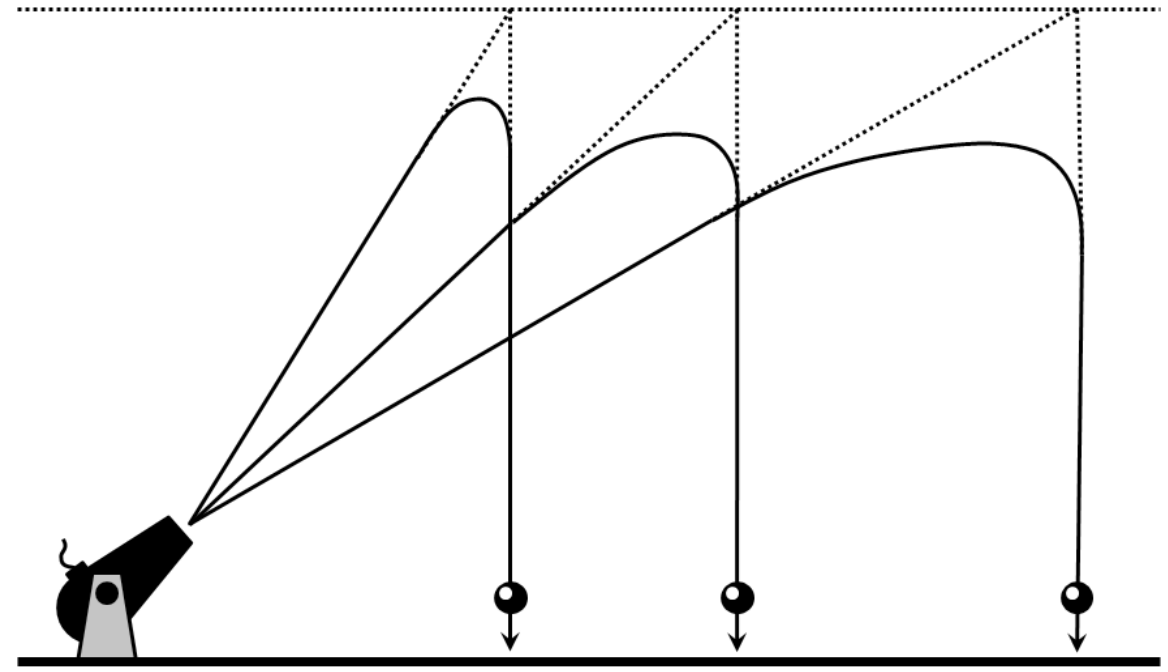

Figura 4. Representação esquemática das trajetórias sugeridas como governando o curso das balas de canhão por Paulus Puchner (1577, cf. Hecht \& Bertamini, 2000).

Os paralelos entre, por um lado, os resultados acerca do Momento Representacional e aqueles obtidos nas tarefas de Física Intuitiva, bem como, por outro, entre esses e as conceções pré-Galilaicas na História da Física, tem sido extensivamente referida na literatura, especialmente por Hubbard (2005) sob a noção de isomorfismo de $2^{\circ}$ grau (Shepard, 1984; Shepard \& Chipman, 1970) - noção que qualifica uma relação funcional (por oposição a estrutural) entre as representações internas e invariantes ecológicos, externos ao observador que, não obstante, adquirem para esse um valor adaptativo (por exemplo, a Gravidade). Neste sentido, a obtenção de um curso temporal para os desfasamentos mnésicos compatível com a trajetória de um projétil com paralelos tanto em tarefas de Física Intuitiva como com conceções proto-científicas da História da Física, atesta a sua prevalência enquanto representação embutida no sistema cognitivo humano. De resto, a emergência desse padrão numa tarefa de localização espacial, na qual, sublinhamos, o participante não tem consciência de estar a indicar um ponto desfasado face ao local de desaparecimento do alvo (aliás, é instruído a ser o mais preciso possível), não só suporta os dados da Física Intuitiva como mostra o papel dessa, enquanto Física Implícita, na perceção de movimento. 


\section{Referências}

Actis-Grosso, R., \& Stucchi, N. (2003). Shifting the start: Backward mislocalization of the initial position of a motion. Journal of Experimental Psychology: Human Perception and Performance, 3, 675-691.

Ashida, H. (2004). Action-specific extrapolation of target motion in human visual system. Neuropsychologia, 42, 1515-1524.

Bertamini, M. (1993). Memory for position and dynamic representations. Memory \& Cognition, 21, 449-457.

Bertamini, M., Spooner, A., \& Hecht, H. (2004). The representation of naïve knowledge about physics. In G. Malcolm (Ed.), Multidisciplinary approaches to visual representations and interpretations. Liverpool, UK: Elsevier.

Brouwer, A. M., Franz, V. H., \& Thornton, I. M. (2004). Representational momentum in perception and grasping: Translating versus transforming object. Journal of Vision, 4, 575-584.

De Sá Teixeira, N. A., Oliveira, A. M., \& Amorim, M.-A. (2010). Combined effects of mass and velocity on forward displacement and phenomenological ratings: A functional measurement approach to the momentum metaphor. Psicologica, 31, 659-676.

Finke, R. A., Freyd, J. J., \& Shyi, G. C. W. (1986). Implied velocity and acceleration induce transformations of visual memory. Journal of Experimental Psychology: General, 115, 175-188.

Freyd, J. J. (1983). The mental representation of movement when static stimuli are viewed. Perception \& Psychophysics, 33, 575-581.

Freyd, J. J. (1987). Dynamic mental representations. Psychological Review, 94, 427-438.

Freyd, J. J. (1992). Dynamic representations guiding adaptive behavior. In F. Macar, V. Pouthas, \& W. J. Friedman (Eds.), Time, action, and cognition: Towards bridging the gap (pp. 309-323). Dordrecht, Netherlands: Kluver Academic.

Freyd, J. J. (1993). Five hunches about percetual processes and dynamic representations. In D. Meyer \& S. Kornblum (Eds.), Attention and performance XIV: Synergies in experimental psychology, artificial intelligence, and cognitive neuroscience. Cambridge, MA: Massachusetts Institute of Technology Press.

Freyd, J. J., \& Finke, R. A. (1984). Representational momentum. Journal of Experimental Psychology: Learning, Memory, and Cognition, 10, 126-132.

Freyd, J. J., \& Finke, R. A. (1985). A velocity effect for representational momentum. Bulletin of the Psychonomic Society, 23, 443-446.

Freyd, J. J., \& Johnson, J. Q. (1987). Probing the time course of representational momentum. Journal of Experimental Psychology: Learning, Memory, and Cognition, 13, 259-269.

Freyd, J. J., Kelly, M. H., \& DeKay, M. L. (1990). Representational momentum in memory for pitch. Journal of Experimental Psychology: Learning, Memory and Cognition, 6, 1107-1117.

Freyd, J. J., Pantzer, T. M., \& Cheng, J. L. (1988). Representing statics as forces in equilibrium. Journal of Experimental Psychology: General, 117, 395-407.

Gentner, D. (2002). Mental models, Psychology of. In N. J. Smelser \& P. B. Bates (Eds.), International Encyclopedia of the Social and Behavioral Sciences (pp. 9683-9687). Amsterdam, Netherlands: Elsevier Science.

Getzmann, S. (2005). Representational momentum in spatial hearing does not depend on eye movements. Experimental Brain Research, 165, 229-238.

Gibson, J. J. (1986). The Ecological Approach to Visual Perception. Hillsdale, NJ: Lawerence Erlbaum.
Gray, R., \& Thornton, I. M. (2001). Exploring the link between time to collision and representational momentum. Perception, 30, 1007-1022.

Grush, R. (2004). The emulation theory of representation: Motor control, imagery, and perception. Behavioral and Brain Sciences, 27, 377-442.

Hecht, H. (2001). Regularities of the physical world and the absence of their internalization. Behavioral and Brain Sciences, 24, 608-617.

Hecht, H., \& Bertamini, M. (2000). Understanding Projectile Acceleration. Journal of Experimental Psychology: Human Perception and Performance, 26, 730-746.

Hubbard, T. L. (1990). Cognitive representation of linear motion: Possible direction and gravity effects in judged displacement Memory \& Cognition, 18, 299-309.

Hubbard, T. L. (1995). Cognitive representation of motion: Evidence for friction and gravity analogues. Journal of Experimental Psychology: Learning, Memory, and Cognition, 21, 241-254.

Hubbard, T. L. (1996). Representational momentum, centripetal force, and curvilinear impetus. Journal of Experimental Psychology: Learning, Memory, and Cognition, 22, 10491060.

Hubbard, T. L. (1997). Target size and displacement along the axis of implied gravitational attraction: Effects of implied weight and evidence of representational gravity. Journal of Experimental Psychology: Learning, Memory, and Cognition, 23, 1484-1493.

Hubbard, T. L. (2001). The effect of height in the picture plane on the forward displacement of ascending and descending targets. Canadian Journal of Experimental Psychology, 55, 325-330.

Hubbard, T. L. (2005). Representational momentum and related displacements in spatial memory: A review of the findings. Psychonomic Bulletin \& Review, 12, 822-851.

Hubbard, T. L., \& Bharucha, J. J. (1988). Judged displacement in apparent vertical and horizontal motion. Perception \& Psychophysics, 44, 211-221.

Hubbard, T. L., Blessum, J. A., \& Ruppel, S. E. (2001). Representational momentum and Michotte's (1946/1963) "Launching Effect" paradigm. Journal of Experimental Psychology: Learning, Memory, and Cognition, 27, 294-301.

Jammer, M. (1957). Concepts of force: A study in the foundations of dynamics. Cambridge, MA: Harvard University Press.

Johansson, G. (1975). Visual motion perception. Scientific American, 232, 76-88.

Kelly, M. H., \& Freyd, J. J. (1987). Explorations of representational momentum. Cognitive Psychology, 19, 369-401.

Kerzel, D. (2000). Eye movements and visible persistence explain the mislocalization of the final position of a moving target. Vision Research, 40, 3703-3715.

Kerzel, D. (2005). Representational momentum beyond internalized physics: Embodied mechanisms of anticipation cause errors of visual short-term memory. Current Directions in Psychological Science, 14, 180-184.

Kerzel, D., \& Gegenfurtner, K. R. (2003). Neuronal processing delays are compensated in the sensorimotor branch of the visual system. Current Biology, 13, 1975-1978.

Koyré, A. (1992). Estudos galilaicos. Lisboa, Portugal: Dom Quixote.

McCloskey, M. (1983). Intuitive Physics. Scientific American, 248, 114-122.

Milner, A. D., \& Goodale, M. A. (1995). The visual brain in action. Oxford, UK: Oxford University Press. 
Munger, M., Owens, T. R., \& Conway, J. (2005). Are boundary extension and representational momentum related? Visual Cognition, 6, 1041-1056.

Müsseler, J., Stork, S., \& Kerzel, D. (2002). Comparing mislocalizations with moving stimuli: The Fröhlich effect, the flash-lag, and representational momentum. Visual Cognition, 9, 120-138.

Nagai, M., Suganuma, M., Nijhawan, R., Freyd, J. J., Miller, G., \& Watanabe, K. (2010). Conceptual influence on the flash-lag effect and representational momentum. In R. Nijhawan \& B. Khurana (Eds.), Space and time in perception and action (pp. 366-378). New York: Cambridge University Press.

Nijhawan, R. (1994). Motion extrapolation in catching. Nature (London), 370, 256-257.

Nijhawan, R. (2008). Visual prediction: Psychophysics and neurophysiology of compensation for time delays (Target article). Behavioral and Brain Sciences, 31, 179-239.

Reed, C. L., \& Vinson, N. G. (1996). Conceptual effects on representational momentum. Journal of Experimental Psychology: Human Perception and Performance, 22, 839-850.

Runeson, S., \& Frykholm, G. (1983). Kinematic specification of dynamics as an informational basis for person-and-action perception: Expectation, gender recognition, and deceptive intention. Journal of Experimental Psychology: General, $112,585-615$.

Shepard, R. N. (1984). Ecological constraints on internal representation: Resonant kinematics of perceiving, imaging, thinking, and dreaming. Psychological Review, 91, 417-447.

Shepard, R. N., \& Chipman, S. (1970). Second-order isomorphism of internal representations: Shapes of states. Cognitive Psychology, 1, 1-17.

Zago, M., \& Lacquaniti, F. (2005). Cognitive, percetual and action-oriented representations of falling objects. Neuropsychologia 43, 178-188. 